\title{
Advanced Dental Cleaning is Associated with Reduced Risk of COPD Exacerbations - A Randomized Controlled Trial
}

\author{
Josefin Sundh (D) \\ Hanan Tanash ${ }^{2}$ \\ Rahi Arian ${ }^{3}$ \\ Alessandra Neves- \\ Guimaraes (D) ${ }^{3}$ \\ Katrin Broberg ${ }^{4}$ \\ Gustav Lindved (1D $)^{5}$ \\ Timo Kern (D) $^{5}$ \\ Konrad Zych (D) ${ }^{5}$ \\ Henrik Bjørn Nielsen (D) ${ }^{5}$ \\ Anders Halling $\mathbb{D}^{6}$ \\ Bodil Ohlsson (1D ${ }^{7}$ \\ Daniel Jönsson (1D ${ }^{4,8,9}$

\begin{abstract}
'Department of Respiratory Medicine, Faculty of Medicine and Health, Örebro University, Örebro, Sweden; ${ }^{2}$ Department of Respiratory Medicine, Skåne University Hospital, Lund University, Malmö, Sweden; ${ }^{3}$ Department of Periodontology and Implantology, Public Dental Service, Örebro, Sweden; ${ }^{4}$ Public Dental Service of Skåne, Lund, Sweden; ${ }^{5}$ Clinical Microbiomics, Copenhagen, Denmark; ${ }^{6}$ Center for Primary Health Care Research, Department of Clinical Sciences Malmö, Lund University, Malmö, Sweden; ${ }^{7}$ Department of Internal Medicine, Skåne University Hospital, Lund University, Malmö, Sweden; ${ }^{8}$ Hypertension and Cardiovascular Disease, Department of Clinical Sciences in Malmö, Lund University, Malmö, Sweden; ${ }^{9}$ Faculty of Odontology, Malmö University, Malmö, Sweden
\end{abstract}

Correspondence: Josefin Sundh Department of Respiratory Medicine, Faculty of Medicine and Health, Örebro University, Örebro, 70185, Sweden

Tel +36702349517

Fax +46I9-186526

Email josefin.sundh@oru.se
Purpose: Infections from the oral microbiome may lead to exacerbations of chronic obstructive pulmonary disease (COPD). We investigated whether advanced dental cleaning could reduce exacerbation frequency. Secondary outcomes were disease-specific health status, lung function, and whether the bacterial load and composition of plaque microbiome at baseline were associated with a difference in outcomes.

Patients and Methods: One-hundred-one primary and secondary care patients with COPD were randomized to intervention with advanced dental cleaning or to dental examination only, repeated after six months. At baseline and at 12 months, data of exacerbations, lung function, COPD Assessment Test (CAT) score, and periodontal status were collected from questionnaires, record review, and periodontal examination. Student's $t$-test and MannWhitney-U (MWU) test compared changes in outcomes. The primary outcome variable was also assessed using multivariable linear regression with adjustment for potential confounders. Microbiome analyses of plaque samples taken at baseline were performed using Wilcoxon signed ranks tests for calculation of alpha diversity, per mutational multivariate analysis of variance for beta diversity, and receiver operating characteristic curves for prediction of outcomes based on machine learning models.

Results: In the MWU test, the annual exacerbation frequency was significantly reduced in patients previously experiencing frequent exacerbations $(p=0.020)$ and in those with repeated advanced dental cleaning $(\mathrm{p}=0.039)$ compared with the non-treated control group, but not in the total population including both patients with a single and repeated visits $(p=0.207)$. The result was confirmed in multivariable linear regression, where the risk of new exacerbations was significantly lower in patients both in the intention to treat analysis (regression coefficient $0.36(95 \%$ CI $0.25-0.52), \mathrm{p}<0.0001)$ and in the population with repeated dental cleaning $(0.16(0.10-0.27), \mathrm{p}<0.0001)$. The composition of microbiome at baseline was moderately predictive of an increased risk of worsened health status at 12 months $(\mathrm{AUC}=0.723)$.

Conclusion: Advanced dental cleaning is associated with a reduced frequency of COPD exacerbations. Regular periodontal examination and dental cleaning may be of clinical importance to prevent COPD exacerbations.

Keywords: alpha diversity, periodontal disease, beta diversity, health status, lung function, plaque microbiome

\section{Introduction}

Exacerbations of chronic obstructive pulmonary disease (COPD) lead to disease progression, re-exacerbations, and increased risk of mortality. ${ }^{1-3}$ Prevention of 
exacerbations includes smoking cessation, ${ }^{4}$ pharmacological maintenance treatment, ${ }^{5-8}$ rehabilitation, ${ }^{9}$ vaccinations, ${ }^{10}$ and structured integrated care. ${ }^{11,12}$ However, recurrent exacerbations are still a major clinical problem associated with lower quality of life ${ }^{13}$ and high healthcare costs. ${ }^{14}$

COPD exacerbations are often caused by bacteria, ${ }^{3}$ acquired from the environment or from colonization of the upper airways. ${ }^{3}$ The oral microbiome, especially that of dental plaque, has been hypothesized to be an important source of infection. The concentration of antibiotics needed to inhibit bacterial strains in steady-state biofilms in vitro is up to 250 times that sufficient for planktonic cultures, and a course of antibiotic treatment for an airway infection seldom eradicates pathogens in dental plaque. $^{15}$ Two small pilot studies have suggested that advanced dental cleaning may reduce the risk of exacerbations of COPD. ${ }^{16,17}$

The primary aim of this study was to determine whether advanced dental cleaning can reduce COPD exacerbation frequency. Secondary aims were to investigate if dental cleaning is associated with improved health status, lung function, or periodontal status and whether specific components of the plaque microbiome at baseline are associated with change in exacerbation frequency.

\section{Methods}

\section{Study Design}

This was a randomized controlled trial, un-blinded for participants but blinded for the researchers who assessed the outcomes and performed the statistical analyses.

\section{Baseline Data Collection}

Inclusion criteria were a recorded diagnosis of COPD (ICD J44). Patients were recruited consecutively from 2016 through 2018 from clinical practice at the Department of Respiratory Medicine at the University Hospital, Örebro; at the Department of Respiratory Medicine at Skåne University Hospital, Malmö; and at several primary healthcare centers in the region Skåne, Sweden (Figure 1). The COPD diagnoses were confirmed by spirometry showing a ratio of forced expiratory volume in one second (FEV1) and forced vital capacity (FVC) below $0.70($ FEV1/FVC $<0.70) .{ }^{18}$ Patients without teeth

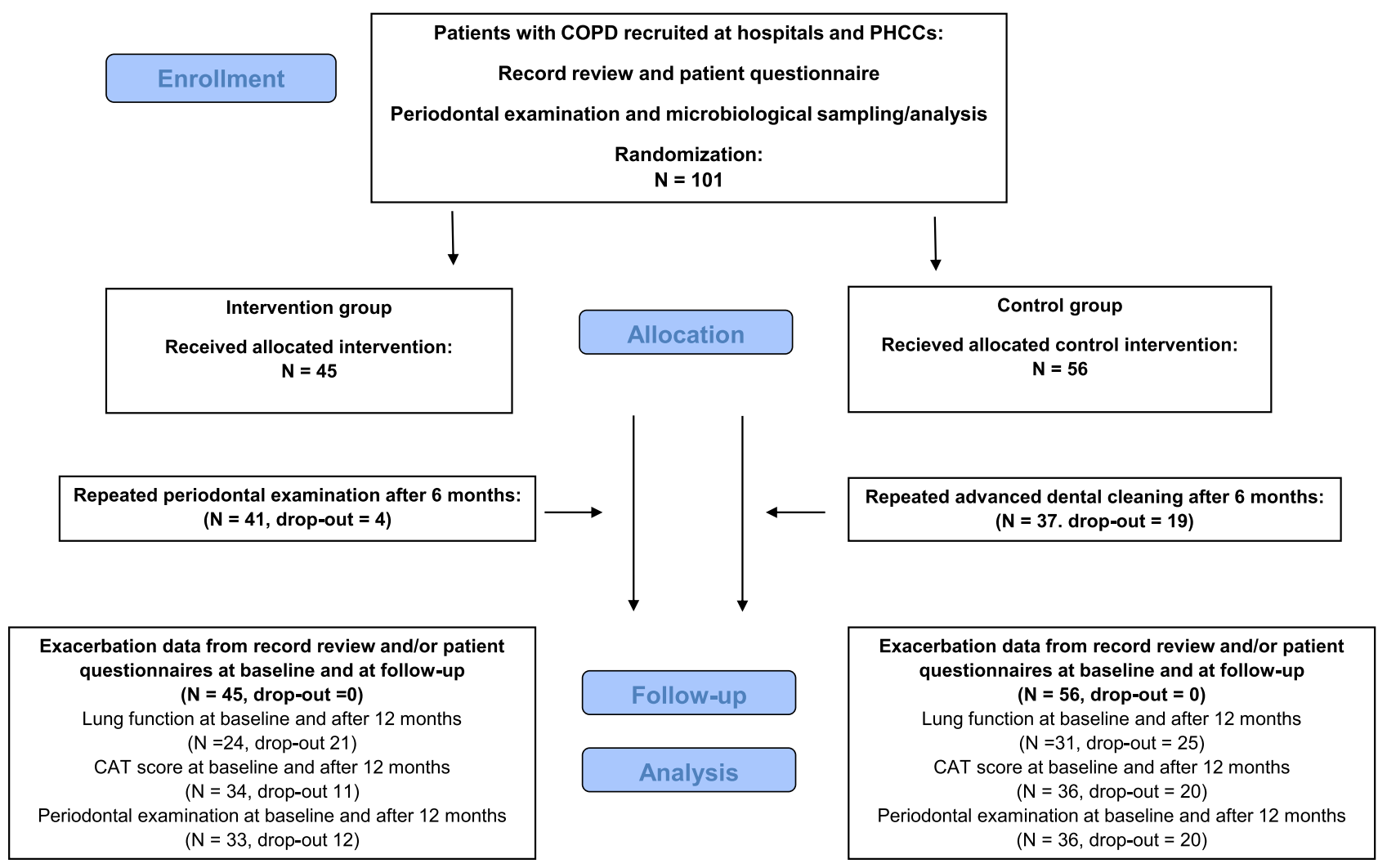

Figure I Flow chart. Adapted CONSORT flow chart of data collection, randomization, and follow-up. Abbreviations: CAT, COPD Assessment Test; PHCC, primary healthcare center. 
or with implants, current lung cancer, or cognitive or linguistic inability to take part in the study were excluded.

All included patients underwent a baseline visit at the Clinic for Dental Care in Skåne (Malmö and Lund) or in Örebro, during which they completed a questionnaire and underwent periodontal examination and sampling of dental plaque for microbiological analysis. The questionnaire included questions on sex, age, smoking status and exacerbation history of the previous 12 months. Subjects also completed the Swedish version of the COPD Assessment Test (CAT), a disease-specific health status instrument based on the subjective assessment of eight items. The score ranges from zero to 40 , with higher scores indicating worse health status. ${ }^{18}$ An exacerbation was defined as a worsening of symptoms resulting in a course of oral antibiotics or steroids. ${ }^{19}$ A complementary record review was performed by the responsible physician to obtain information on height, weight, comorbidities, maintenance treatment, exacerbation history, and spirometry data.

Periodontal examinations were performed on six sites per tooth, including the third molar. Inter-examiner agreement was set at $\geq 95 \%$ within $\pm 1 \mathrm{~mm}$ probing depth. Data of bleeding upon probing, periodontal probing depth, and attachment loss were recorded. Severe periodontal disease was defined, in accordance with the American Academy of Periodontology and the US Centers for Disease Control and Prevention definitions at the time of planning the study, by number of periodontal pockets $>5 \mathrm{~mm} \cdot{ }^{20}$ Microbiological sampling of dental plaque was performed at baseline and after 12 months by scraping the sulcus of the mesio-lingual surface of teeth $32,31,41$, and 42 using a curette. The plaque samples were pooled by immersing the curette in a buffer optimized for extracting high-quality 16S rRNA (DNA Genotek, OMNIgene OMR-110).

\section{Randomization and Intervention}

A power analysis based on the standard deviations from the pilot study of Kucukoskun et $\mathrm{al}^{16}$ indicated $80 \%$ power to detect an overall difference of $p<0.05$ of 1.5 exacerbations per year in a sample of 49 subjects in each group. In the referred study, the mean number and standard deviations of exacerbation frequency after one year decreased from $3 \pm 1.83$ to $1.95 \pm 1.46(\mathrm{p}=0.01)$ in the intervention group and was unchanged in the control group $(3.5 \pm 4.62$ vs $3.25 \pm 3.35, \mathrm{p}=0.48$ ).

At the Clinic for Dental Care, the patients were randomized 1:1, by blocks of ten, to intervention with advanced dental cleaning or to a control group. The randomization was performed online using GraphPad. The intervention was a modification of the full-mouth disinfection protocol, performed at baseline and after 6 months. ${ }^{21}$ If needed to complete the baseline intervention, the advanced dental cleaning was divided into more than one occasion within a short time period. The oral biofilm was disrupted and removed on the sub- and supra-gingival surfaces of the teeth by scaling, with the supra-gingival surface biofilm disrupted using the prophylactic paste RDA 250 supplemented with curettage and ultrasonic debridement. The tongue was cleaned using a tongue scraper while holding the patients tongue with a piece of gauze. Finally, the patient was instructed to rinse with $10 \mathrm{~mL}$ of $2 \mathrm{mg} / \mathrm{mL}$ chlorhexidine mouthwash for one minute, at the visit and once per day for the subsequent seven days, and were given oral hygiene instructions. The control group underwent an examination and supra-gingival cleaning using toothpaste, corresponding to tooth brushing.

\section{Follow-Up}

All patients were called to return to the Clinic for Dental Care after six and 12 months for periodontal examination and a follow-up interview. At the six-month visit, the advanced dental cleaning was repeated in the intervention group. At the 12-month visit, both the intervention and control groups were given the advanced dental cleaning. The incidence of adverse events was noted in both groups. After 12 months, records of all patient who underwent the baseline visit or intervention were reviewed for repeated spirometry and exacerbations regardless of whether they returned for follow-up.

\section{Analysis of Microbiota}

The microbiological and statistical analyses of plaque samples were performed at Clinical Microbiomics, Copenhagen. DNA was extracted using NucleoSpin ${ }^{\circledR} 96$ Soil (Macherey-Nagel). Polymerase chain reaction was conducted using the forward primer S-D-Bact-0341b-S-17 and reverse primer S-D-Bact-0785-A-A-21 with Illumina adapters attached. ${ }^{22}$ The samples were sequenced in an Illumina MiSeq sequencer using the MiSeq Reagent Kit V3 (Illumina) for $2 \times 300$ bp paired-end sequencing, and an adjusted dada 2 pipeline processed the sequence data into the amplicon sequence variant (ASV) abundance table. ${ }^{23}$ Taxonomic assignment of the detected ASVs was conducted using a naïve Bayesian classifier algorithm comparing the ASV sequences to the expanded Human Oral Microbiome Database (eHOMD v.15.21). ${ }^{24}$ 
Microbiological richness (alpha diversity) was assessed as observed richness (number of ASVs present in a sample) and the Shannon index (ASV composition). Dissimilarity in the taxonomic composition among samples (beta diversity) was calculated as Bray-Curtis dissimilarity.

A proprietary machine learning pipeline based on the $\mathrm{R}$ package SIAMCAT ${ }^{25}$ was used to infer associations between ASVs from the baseline samples and differences in exacerbation frequency and CAT score at the 12-month follow-up visit from those at baseline.

\section{Variables}

Baseline variables included sex, age, smoking status, body mass index (BMI), comorbidities, maintenance COPD treatment, total number of exacerbations and hospitalized exacerbations the previous year, CAT score, FEV1 in percentage of predicted value (FEV1\%pred), and number of periodontal pockets $>5 \mathrm{~mm}$.

Smoking status was categorized as never, former, and current daily smoking. BMI was categorized as $<22.0$, 22.0-29.9, and $>30.0 \mathrm{~kg} / \mathrm{m}^{2} .{ }^{26}$ Maintenance treatment was categorized as none, long-acting bronchodilation with long-acting muscarinic antagonists and/or longacting beta-2-agonists, or any combination including inhaled corticosteroids or roflumilast.

Severity of disease was presented both as Global Initiative on Obstructive Lung Disease (GOLD) group (A-D) and stage (1-4). ${ }^{19}$ According to GOLD, a CAT score $>10$ corresponds to a severe symptom level, and frequent exacerbations is defined as $>2$ exacerbations or $>$ one hospitalized exacerbation in one year. GOLD group A denotes a low level of symptoms and no frequent exacerbations, group B a high level of symptoms and no frequent exacerbations, group $\mathrm{C}$ a low level of symptoms and frequent exacerbations, and group $\mathrm{D}$ high level of symptoms and frequent exacerbations. GOLD stage 1 denotes FEV1\%pred $>80$, stage 2 is FEV1\%pred 50-79, stage 3 is FEV1\%pred $30-49$, and stage 4 is FEV1\% pred $<30$.

\section{Statistical Analysis}

Statistical analyses were performed using SPSS version 25 (IBM Corp, Armonk, NY). The primary outcome variable, difference in exacerbation frequency, was calculated as the difference between the number of exacerbations in the 12 months preceding inclusion and during the follow-up period of 12 months. The secondary outcome variables were differences in CAT score, FEV1\%pred, and number of periodontal pockets $>5 \mathrm{~mm}$ between baseline and the 12 month follow-up visit. Kolmogorov-Smirnov and ShapiroWilk tests were used to test the null hypothesis of normal distribution for continuous baseline and outcome variables.

Baseline and outcome variables in the intervention and control groups were compared using cross-tabulation and chi-squared test for categorical variables. Student's $t$-test was used for normally distributed continuous variables (age, FEV1\%pred, and CAT score) and Mann-Whitney$\mathrm{U}$ (MWU) test for non-normally distributed continuous variables (exacerbation frequency and number of pockets $>5 \mathrm{~mm}$ ).

Comparisons were conducted as intention-to-treat analysis of all randomized patients $(n=101)$. Subgroup analyses were performed within the population returning for the six-month follow-up visit $(n=78)$. The analysis of the primary outcome variable was repeated stratified for severity of disease according to GOLD with merging of groups $\mathrm{A}$ and $\mathrm{B}$, groups $\mathrm{C}$ and $\mathrm{D}$, stages 1 and 2, and stages 3 and 4 , and for presence or absence of severe periodontal disease. The association of intervention group with the primary outcome variable was also tested in a multivariable Poisson regression analysis, with adjustment for sex, age, smoking, CAT score, exacerbations frequency and FEV1\% predicted at baseline. The potential confounding factors were chosen a priori based on previous knowledge, ${ }^{19}$ and were tested in univariable Poisson regression analyses. In addition, a paired test of the effect of intervention on number of pockets per subject within each randomization group was performed using the Wilcoxon signed rank test.

An attrition analysis compared baseline characteristics of patients attending and those declining the six-month follow-up visit, and the main analyses were repeated including patients with complete lung function data at baseline.

The Wilcoxon signed rank test was used to calculate differences in alpha-diversity, and per mutational multivariate analysis of variance to investigate differences in beta-diversity within and between the intervention and control groups at baseline and 12-month follow-up. In the machine learning analyses, Least Absolute Shrinkage and Selection Operator models ${ }^{27}$ of the baseline microbiome composition data were used to predict change in exacerbation frequency and CAT score, measured both as continuous and binary (decreased vs increased/unchanged) outcome variables. The predictive ability was evaluated using receiver operating characteristic curves. An area 
under curve (AUC) value $>0.9$ was considered excellent, 0.8-0.89 good, 0.7-0.79 fair/modest, and 0.5-0.69 poor/ marginal. $^{28}$ In all other analyses, $\mathrm{p}<0.05$ was regarded as significant.

\section{Ethics}

The study was conducted in accordance with the Declaration of Helsinki. All participants gave written informed consent before the study start. The study was approved by the Ethical Board in Lund (Dnr 2015/625). The study protocol was published at clinicaltrials.gov (Id nr NCT02619903).

\section{Results}

\section{Patient Characteristics and Baseline Data}

A total of 101 patients were included, randomized at the first dental clinic visit and followed up after 12 months with collection of data on exacerbations. Seventy-eight of these returned for a second periodontal examination/ advanced dental cleaning after six months, and 69 of those attended the 12-month follow-up. More than twothirds of the patients were recruited from secondary care, and more than half experienced two or more exacerbations during the 12 months prior to inclusion (Table 1). The most common comorbidities were hypertension, diabetes mellitus, and cardiovascular disease. No significant differences were found in patient characteristics between the randomization groups at baseline, either in the main population (Table 1) or in the subpopulation of patients attending the six-month follow-up visit (data not shown).

The main reasons for declining to participate in the study were lack of teeth or having dental implants and unwillingness to see a dental practitioner other than their regular provider. The major reason for dropout at the follow-up visits was the inconvenience of extra visits. The attrition analysis showed no significant differences in sex, age, comorbidities, maintenance treatment, previous exacerbation frequency, or COPD severity with respect to completion of the full study protocol. Repeating the main analyses in only those patients in whom spirometry confirming the COPD diagnosis was completed at baseline did not substantially change the results (data not shown).

\section{Effect of Intervention}

In the intention to treat analysis, exacerbation frequency at the 12-month follow-up was slightly decreased compared to baseline in the group randomized to intervention but not in the control group, but did not reach significance. In the per protocol analysis of the subpopulation that underwent a repeated advanced dental cleaning or examination at six months, the annual exacerbation frequency decreased significantly in the intervention group compared with controls (Table 2$)$. In the small group $(n=23)$ with a baseline visit but no 6-month visit, no statistically significant difference in exacerbation frequency was found (change in exacerbation frequency (median -0.5 (IQR $-2.0-0)$ vs median -1.5 (IQR $-3.5-0.75), \mathrm{p}=0.264)$ ).

The unadjusted Poison regression analyses showed that the independent variable intervention group, as well as all potential confounders, were associated with a risk of exacerbations during the follow-up period (data not shown). The adjusted Poisson analysis showed that being in the intervention group with advanced dental cleaning was independently associated with a significantly lower risk of exacerbations during the follow-up period, both in the main group and in the subgroup with repeated intervention (Table 3).

No significant differences in secondary outcomes between randomization groups were found in the total population or in the subpopulation attending the sixmonth follow-up visit (Table 2). Among subjects with deep periodontal pockets at baseline, the number of deep periodontal pockets was reduced in the intervention group $(p=0.044)$, but not in the control group $(p=0.360)$.

In the stratified analyses, the annual exacerbation frequency was significantly reduced in the intervention group for patients categorized as GOLD group C or D. In the subpopulation with repeated examination/intervention at six months, the annual exacerbation frequency was significantly reduced in both GOLD group $\mathrm{A} / \mathrm{B}$, group $\mathrm{C} / \mathrm{D}$ and stage $3 / 4$ (Table 4 ). In patients with no deep periodontal pockets, there was decreased exacerbation frequency both in the total and subpopulation (Table 4). No major adverse events of periodontal disease were recorded during the study period.

\section{Microbiota Analyses}

DNA extracted from dental plaque was $16 \mathrm{~S}$ gene sequenced, and more than $90 \%$ of the high-quality microbiome reads in the samples could be assigned to a genus and over $60 \%$ to a species. No significant changes were observed in dental plaque microbiome alpha or beta diversity, either between groups or within each group (Figures 2 and 3). The machine learning model showed limited 
Table I Patient Characteristics at Baseline

\begin{tabular}{|c|c|c|c|c|}
\hline \multirow[t]{2}{*}{ Baseline Data } & All & Intervention & Control & \multirow[t]{2}{*}{ p-value } \\
\hline & $\mathbf{N}=|\mathbf{0}|$ & $N=45$ & $\mathbf{N}=\mathbf{5 6}$ & \\
\hline \multicolumn{5}{|l|}{ Level of care } \\
\hline Primary care & $3 I(3 \mid)$ & $13(29)$ & $18(32)$ & 0.725 \\
\hline Secondary care & $70(69)$ & $32(7 \mathrm{I})$ & $38(68)$ & \\
\hline \multicolumn{5}{|l|}{ Sex } \\
\hline Male & $40(40)$ & $17(38)$ & $23(4 I)$ & 0.737 \\
\hline Female & $61(60)$ & $28(62)$ & $33(59)$ & \\
\hline Age (years) & $69.4+8.6$ & $68.3+8.4$ & $70.3+8.7$ & 0.248 \\
\hline \multicolumn{5}{|l|}{ Smoking status } \\
\hline Current & $25(25)$ & $10(22)$ & $15(27)$ & 0.718 \\
\hline Ex & $69(68)$ & $31(69)$ & $38(68)$ & \\
\hline Never & $7(7)$ & $4(9)$ & $3(5)$ & \\
\hline \multicolumn{5}{|l|}{ BMI $(\mathbf{k g} / \mathrm{m} 2)^{*}$} \\
\hline$<22.0$ & $19(19)$ & II (25) & $8(14)$ & 0.385 \\
\hline 22.0 to 29.9 & $65(65)$ & $26(59)$ & $39(70)$ & \\
\hline$\geq 30.0$ & $16(16)$ & $7(16)$ & $9(16)$ & \\
\hline \multicolumn{5}{|l|}{ Comorbidity } \\
\hline Hypertension & $36(36)$ & $17(38)$ & $19(34)$ & 0.688 \\
\hline Cardiovascular disease & $32(32)$ & $16(36)$ & $16(29)$ & 0.453 \\
\hline Diabetes mellitus & $13(13)$ & $6(13)$ & $7(13)$ & 0.901 \\
\hline \multicolumn{5}{|l|}{ Maintenance treatment } \\
\hline None & $34(34)$ & $17(38)$ & $17(30)$ & 0.433 \\
\hline LABA and/or LAMA & $2 I(2 I)$ & $7(16)$ & $14(25)$ & 0.245 \\
\hline ICS combinations or roflumilast & $46(46)$ & $22(49)$ & $24(43)$ & 0.545 \\
\hline FEVI\%pred** & $47.9 \pm 17.9$ & $48.4 \pm 18.6$ & $47.5 \pm 17.5$ & 0.832 \\
\hline CAT score ${ }^{* * *}$ & $18.2 \pm 8.6$ & $17.4 \pm 9.1$ & $18.9 \pm 8.3$ & 0.405 \\
\hline $\begin{array}{l}\text { Number of exacerbations } \\
\text { previous } 12 \text { months }\end{array}$ & $2.0(0.5-3.0)$ & $2.0(0.5-3.0)$ & $2.0(0.3-3.0)$ & 0.532 \\
\hline \multicolumn{5}{|l|}{ GOLD stages** } \\
\hline 1 & $3(4)$ & $2(5)$ & I (2) & 0.107 \\
\hline 2 & $28(36)$ & $18(49)$ & $28(36)$ & \\
\hline 3 & $32(4 I)$ & $12(32)$ & $32(4 I)$ & \\
\hline 4 & $15(19)$ & $5(14)$ & $15(19)$ & \\
\hline \multicolumn{5}{|l|}{ GOLD groups**** } \\
\hline A & $9(9)$ & $6(13)$ & $3(5)$ & 0.470 \\
\hline B & $24(24)$ & $10(22)$ & $14(26)$ & \\
\hline C & $9(9)$ & $5(I I)$ & $4(7)$ & \\
\hline $\mathrm{D}$ & $58(58)$ & $24(53)$ & $34(62)$ & \\
\hline Number of pockets $>5 \mathrm{~mm}$ & $1.0(0.0-3.5)$ & $1.0(0.0-3.0)$ & $1.0(0.0-4.0)$ & 0.826 \\
\hline Severe periodontal disease & $28(27.7)$ & $15(33.3)$ & $13(23.2)$ & 0.273 \\
\hline
\end{tabular}

Notes: Patient characteristics at baseline. Cardiovascular disease included a history of ischemic heart disease, heart failure, atrial fibrillation, and cerebrovascular disease. Data presented as numbers (percentages), mean \pm standard deviations or medians (interquartile ranges), and analyzed using chi-squared test, $t$-test, or Mann-Whitney$U$ test. *Missing data $\mathrm{n}=1$, **Missing data $\mathrm{n}=23$, ***Missing data $\mathrm{n}=1$.

Abbreviations: BMI, body mass index; CAT, COPD Assessment Test; FEV $\%$ pred, forced expiratory volume in one second in percentage of predicted value; GOLD, Global Initiative for Obstructive Lung Disease; ICS, inhaled corticosteroids; LAMA, long-acting muscarinic antagonists; LABA, long-acting beta-2-agonists. 
Table 2 Effect of Advanced Dental Cleaning on Primary and Secondary Outcomes (MWU Test)

\begin{tabular}{|c|c|c|c|}
\hline & Intervention & Control & p-value \\
\hline & $N=45$ & $N=56$ & \\
\hline $\begin{array}{l}\text { Change in annual exacerbation frequency } \\
\text { Total population, } \mathrm{n}=10 \mathrm{I} \\
\text { Population attending 6-month follow-up, } \mathrm{n}=78\end{array}$ & $\begin{array}{l}-1.0(-3.0-0.0) \\
-1.0(-3.5-0.0)\end{array}$ & $\begin{array}{l}0.0(-2.0-0.0) \\
0.0(-1.0-1.0)\end{array}$ & $\begin{array}{l}0.207 \\
0.039\end{array}$ \\
\hline $\begin{array}{l}\text { Change in CAT score } \\
\text { Population attending 6-month follow-up, } n=70\end{array}$ & $-0.7 I \pm 6.44$ & $0.39 \pm 6.07$ & 0.467 \\
\hline $\begin{array}{l}\text { Change in FEVI\%pred } \\
\text { Total population, } n=55 \\
\text { Population attending 6-month follow-up, } n=40\end{array}$ & $\begin{array}{l}-2.42 \pm 8.87 \\
-2.19 \pm 9.43\end{array}$ & $\begin{array}{l}-3.39 \pm 6.07 \\
-2.32+5.20\end{array}$ & $\begin{array}{l}0.649 \\
0.958\end{array}$ \\
\hline $\begin{array}{l}\text { Change in number of periodontal pockets }>5 \mathrm{~mm} \\
\text { Population attending the } 6 \text {-month follow-up, } \mathrm{n}=69\end{array}$ & $0.0(-1.0-0.5)$ & $0.0(-0.75-1.75)$ & 0.305 \\
\hline
\end{tabular}

Notes: Difference between the intervention and control groups in change of annual exacerbation frequency, CAT score, FEVI\%pred, and number of periodontal pockets $>$ $5 \mathrm{~mm}$ from baseline to 12-month follow-up. Values are presented as mean \pm standard deviation or median (interquartile ranges). Analyses performed using $t$-test or MannWhitney-U test.

Abbreviations: CAT, COPD Assessment Test; FEVI\%pred, Forced expiratory volume in one second in percentage of predicted value; $M W U$ test, Mann-Whitney- $U$ test.

predictive capability of oral microbiome for exacerbation frequency and CAT scores as continuous variables. When the measures were binarized, AUC for the baseline microbiome was 0.627 for exacerbation frequency and 0.723 for CAT score (Figure 4).

\section{Discussion}

Our primary finding was that repeated but not a single baseline intervention with advanced dental cleaning reduced exacerbation frequency in patients with COPD. In patients with frequent exacerbations, as in GOLD group

Table 3 Effect of Advanced Dental Cleaning on Primary Outcomes (Poisson Regression)

\begin{tabular}{|c|c|c|c|c|}
\hline \multirow[t]{3}{*}{ Baseline Variables } & Adjusted Analyses & \multirow[t]{3}{*}{ p-value } & Adjusted Analyses & \multirow[t]{3}{*}{ p-value } \\
\hline & Total Population & & Population Attending 6-Month Follow-Up & \\
\hline & Regression Coefficient (95\% Cl) & & Regression Coefficient ( $95 \% \mathrm{Cl}$ ) & \\
\hline \multicolumn{5}{|l|}{ Randomization group } \\
\hline Intervention & $0.36(0.25$ to 0.52$)$ & $<0.0001$ & $0.16(0.10$ to 0.27$)$ & $<0.0001$ \\
\hline Control & Ref & & & Ref \\
\hline \multicolumn{5}{|l|}{ Sex } \\
\hline Male & Ref & $<0.0001$ & Ref & $<0.0001$ \\
\hline Female & 0.32 (0.23 to 0.45$)$ & & $0.32(0.21$ to 0.47$)$ & \\
\hline Age & 0.97 (0.95 to 0.98$)$ & $<0.0001$ & $0.98(0.97$ to 1.00$)$ & 0.061 \\
\hline Current daily smoking & $0.56(0.37$ to 0.88$)$ & 0.011 & $0.4 I(0.23$ to $0.7 I)$ & 0.002 \\
\hline CAT score & 1.07 (1.04 to I.09) & $<0.0001$ & 1.06 (1.03 to 1.10$)$ & $<0.0001$ \\
\hline Exacerbation frequency & I.II (I.04 to I.I7) & 0.001 & 1.25 (I.I4 to I.36) & $<0.0001$ \\
\hline FEVI\%pred & 1.01 ( 1.00 to 1.02$)$ & 0.283 & $1.02(1.01$ to 1.03$)$ & 0.002 \\
\hline
\end{tabular}

Notes: Association of intervention with exacerbations frequency during the study period, compared with the control group, assessed by Poisson regression analysis with adjustment for sex, age, current daily smoking, CAT score, exacerbation frequency previous 12 months, and FEVI\%pred at baseline. The model was repeated both in the total population and in the population attending the 6-month follow-up with repeated intervention.

Abbreviations: CAT, COPD Assessment Test; Cl, confidence interval; FEVI\%pred, Forced expiratory volume in one second in percentage of predicted value. 


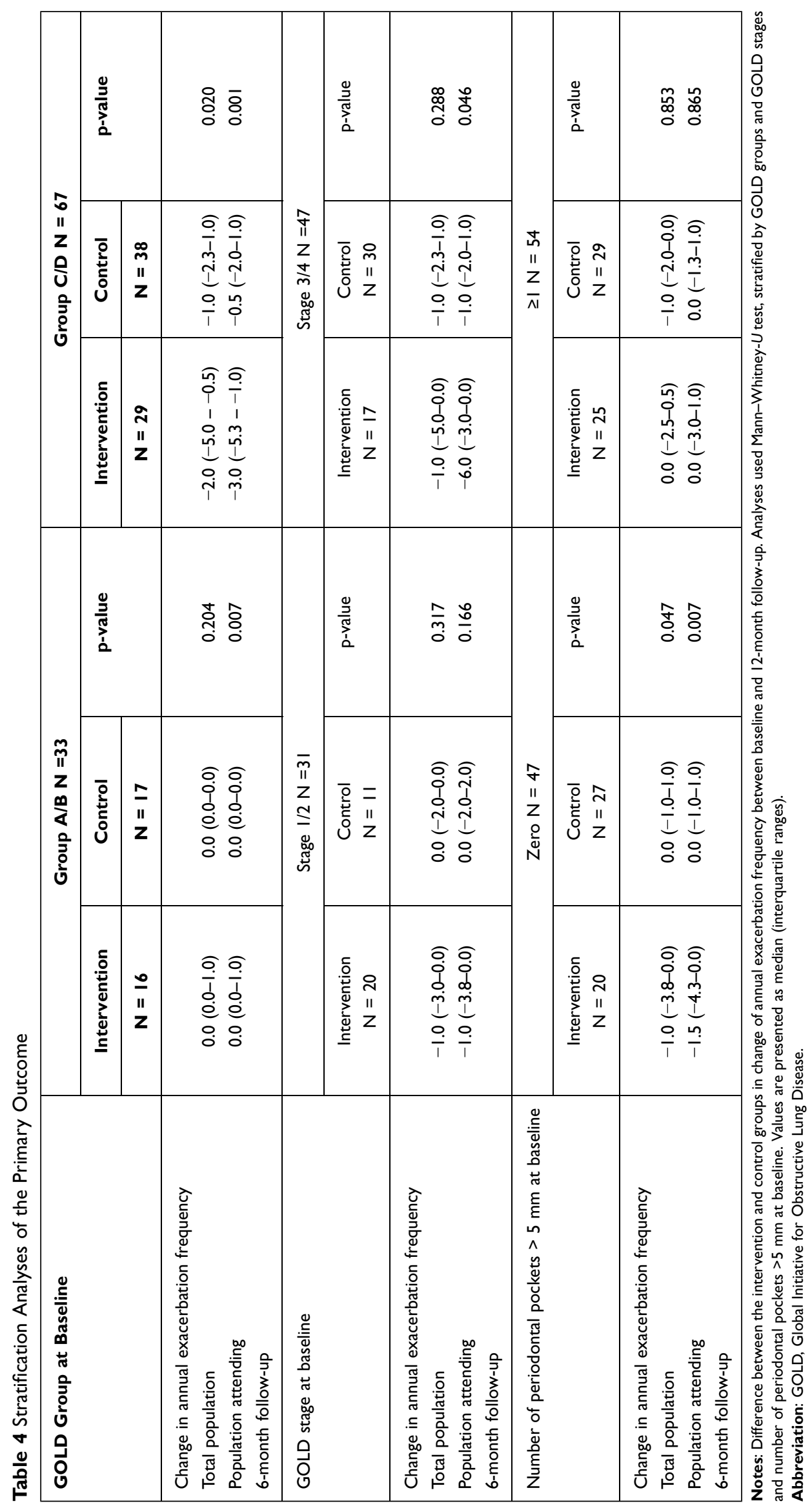




\section{Observed richness}

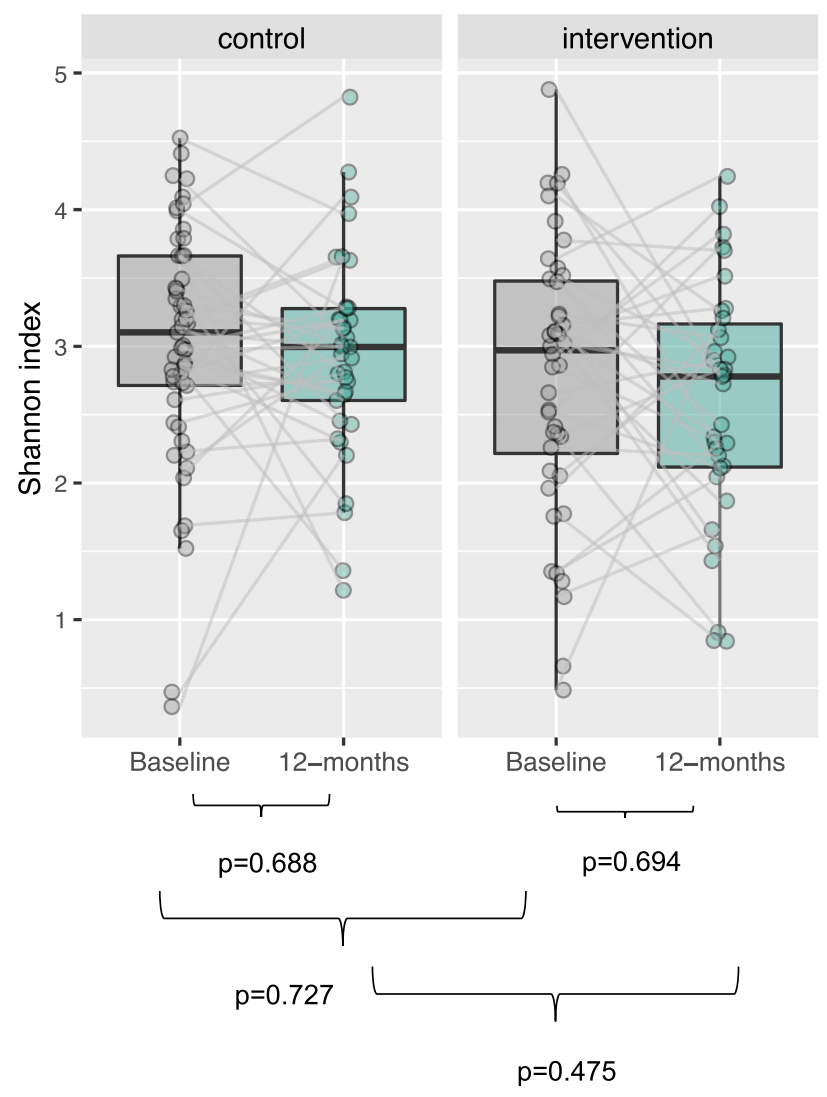

\section{Shannon Index}

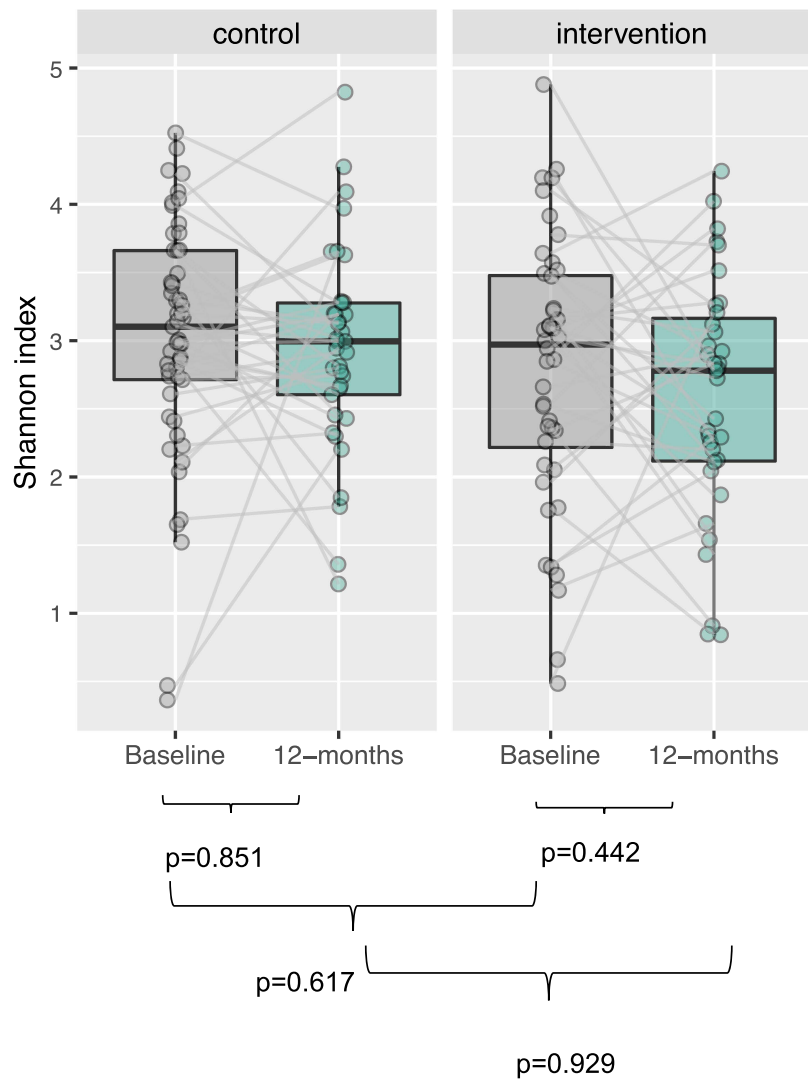

Figure 2 Alpha diversity of microbiome. Alpha diversity as measured by observed richness and Shannon index, with comparison within each randomization group during follow-up and between randomization groups at baseline and at 12-month follow-up using Wilcoxon signed ranks tests.

$\mathrm{C}$ and $\mathrm{D}$, a single advanced dental cleaning was sufficient to result in significant differences in the main outcome between the intervention and the control groups. As the result was confirmed using a multivariable linear regression analysis with adjustment for potential confounders, the reported effect of advanced dental cleaning should be valid and not be explained by random differences between the randomization groups. Abundance of microbiota as measured by ASV could predict a binary change in health status, and, less accurately a binarized change in exacerbation frequency.

Our findings of decreased exacerbation frequency attributed to dental cleaning are in accordance with two smaller randomized controlled studies and several observational studies. ${ }^{17,18,29-31}$ A thorough review by Apessos et $\mathrm{al}^{32}$ reported no previous shown effect of periodontal treatment on health status as measured by Saint Georges Respiratory Questionnaire. Our study suggests that a high microbiological richness seem to be associated with health status as measured by CAT score, although the effect of periodontal intervention in health status is still unclear. Our study extends the evidence to include a broader spectrum of COPD patients with generally low mean FEV1\% pred and, potentially, a more valid outcome using data collected by both patient questionnaires and record reviews.

The results demonstrate that advanced dental cleaning may be of particular benefit to individuals experiencing frequent exacerbations, regardless of the presence of deep periodontal pockets. We chose to call the intervention "advanced dental cleaning", as opposed to "non-surgical periodontal treatment", because the hypothesis of dental plaque being a reservoir for systemic opportunistic pathogens also applies to individuals without periodontal disease, and periodontal treatment implies periodontal disease. This is consistent with the absence of 


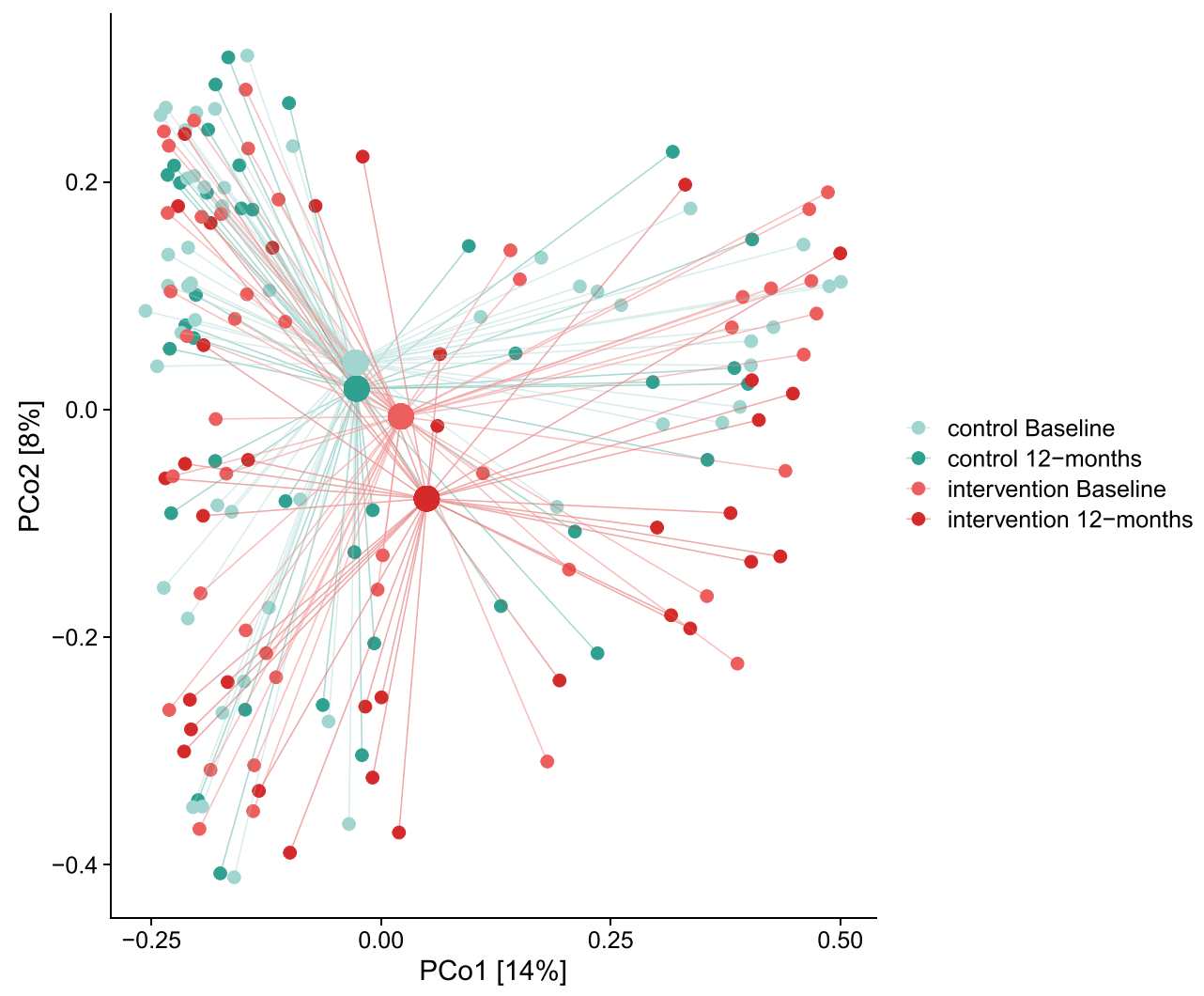

Figure 3 Beta diversity of microbiome. PCoA plot of beta-diversity samples (small dots) and centroids (large dots). Difference in beta diversity based on per mutational multivariate analysis of variance.

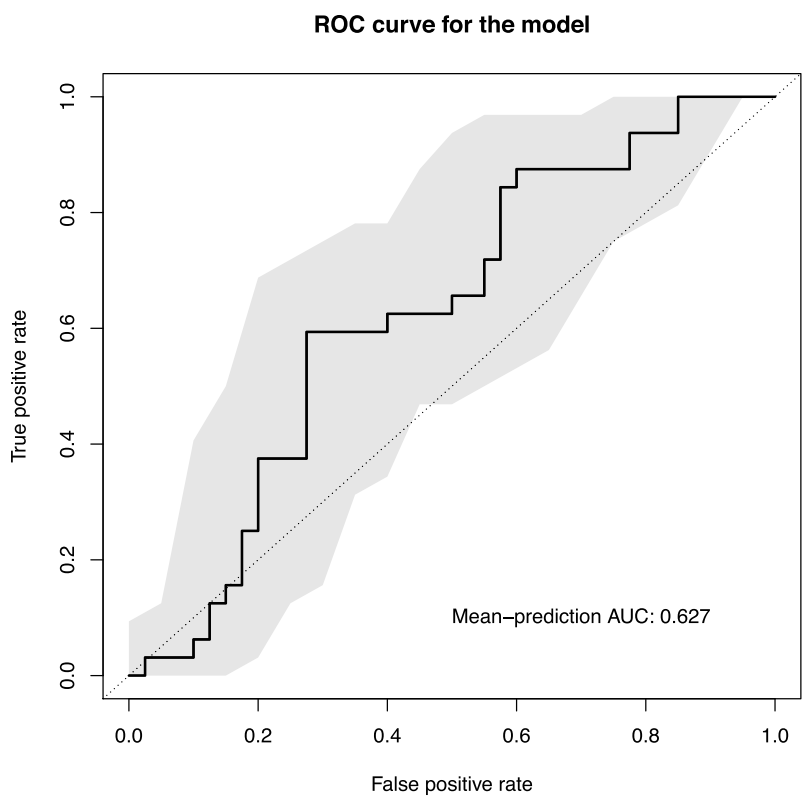

Change in exacerbation frequency

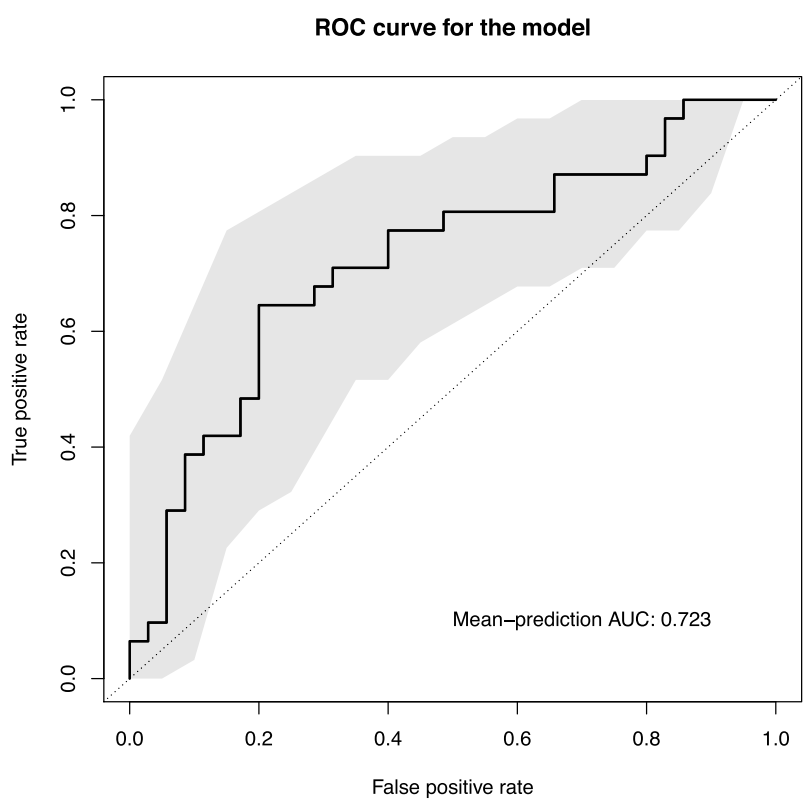

Change in CAT score

Figure 4 Prediction of change in outcomes based on microbiota. Prediction of binarized outcomes from differences in abundance of microbiological features, using machine learning models with ROC-curves.

Abbreviations: CAT, COPD Assessment Test; ROC, Receiver operating characteristic. 
a correlation of the presence of deep periodontal pockets with exacerbations.

In inter-group comparisons, the intervention showed no significant effect on the number of deep periodontal pockets. However, in a paired intra-group comparison, intervention was associated with a reduced number of deep periodontal pockets in subjects with periodontal pockets at baseline. The lack of a substantial effect of intervention on periodontal pockets may be attributed to the frequent use of antibiotics in both groups. ${ }^{33}$

The effect of the intervention with advanced dental cleaning may have different explanations, such as reduction of the overall bacterial burden, a change in bacterial pattern, or just a reduction of general inflammation in the airways.

The microbiota analyses showed a visual tendency towards reduction of microbiological richness in both randomization groups, and towards a more pronounced change in taxonomic composition in the interventional group, but no changes were statistically significant. However, the modest predictive ability of the machine learning model based on microbiome composition at baseline suggests that advanced dental cleaning may reduce the burden of bacteria, consequently influencing symptoms and exacerbations in patients with COPD. The absence of statistically significant differences in alpha and beta diversity between randomization groups and of associations of alpha and beta diversity at baseline with continuous outcome variables, may be a reflection of insufficient power for the microbiome analysis or due to an interventional effect mediated by mechanisms other than microbiological.

Research reviewed by Sczepanik et $\mathrm{al}^{34}$ and Sapey et $\mathrm{al}^{35}$ leads to the hypotheses that both oral microbiota and the host response induce an inflammatory response, for example through release of neutrophilic proteinases, that in itself may contribute to COPD exacerbations. Periodontal treatment may mediate a systemic antiinflammatory effect as shown by alterations in systemic markers of inflammation such as the endothelial profile, CRP, and IL-6. ${ }^{36-39}$ Thus, the systemic anti-inflammatory effect of periodontal disease treatment may constitute an additional mechanism mediating the reduction in COPD exacerbation risk.

The overall decrease in annual exacerbation frequency was significant only after repeated advanced dental cleaning, possibly reflecting a group of patients more likely to follow physician instructions or to a placebo effect among those who followed the protocol. However, the fact that the intention to treat analysis showed a significant effect in patients with previous frequent exacerbations (GOLD C and D) suggests a true effect and that a contributing factor to the absence of a significant effect in the total population could be that some patients did not experience exacerbations.

\section{Strengths and Limitations}

A major strength of the study is the investigation of patient-oriented outcome measures and their association with the oral microbiota. The population included all severity stages of COPD, which should increase the generalizability of results, and the randomized trial design minimized confounding effects.

The major limitation is the dropout of one-third of the study participants, mainly due to inconvenience of additional visits. The drop-out was higher in the control group, which could be explained by the open design. However, data of exacerbations was available for all enrolled patients, and patient characteristics at baseline did not differ for patients dropping out. We speculate that the result of the MWU test in the total population may have been affected by the loss of power due to the drop-outs. As the drop-outs reflect clinical reality, future studies should take this into account when calculating the sample size.

Although alpha and beta diversity of the oral microbiome were analyzed both at baseline and follow-up, currently no machine learning models can take full advantage of the longitudinal design for investigating change in the composition of microbiome with respect to outcomes. Nevertheless, to our knowledge, this is the first machinelearning aided microbiome study of COPD, and the results indicate that oral microbiome data may be useful in prediction models for disease-specific outcomes.

\section{Conclusions}

Advanced dental cleaning is associated with a reduced frequency of COPD exacerbations. Regular periodontal examination and dental cleaning may be of clinical importance to prevent COPD exacerbations. Diagnostic models based on the oral microbiome may have a limited potential for prediction of clinical outcomes in COPD, but needs to be further investigated.

\section{Data Sharing Statement}

Data cannot be made freely available as they are subject to privacy in accordance with the Swedish Public Access to Information and Secrecy Act but can be provided to researchers upon request, subject to a review of privacy. Requests for data can be sent to the corresponding author. 


\section{Acknowledgments}

We thank Bodil Roth for excellent help with the logistics of the study and Susanne Rundqvist and the rest of the staff at the Public Dental Service in Skåne and Örebro for examining, randomizing, and treating the patients. We are also grateful for help with recruitment at the Departments of Respiratory Medicine in Malmö and Örebro, as well as the health centers Örestadskliniken (respiratory nurse May Bugge), Kroksbäckskliniken (respiratory nurse Hoxhaj Rama Teoreta), Viktoria Park, and Ellenbogen.

\section{Funding}

The study was founded by grants from Oral Health Related Research by Region Skåne and the Swedish Dental Public Service of Skåne.

\section{Disclosure}

The authors report no conflicts of interest in this work.

\section{References}

1. Donaldson GC, Seemungal TA, Bhowmik A, et al. Relationship between exacerbation frequency and lung function decline in chronic obstructive pulmonary disease. Thorax. 2002;57(10):847-852. doi:10.1136/thorax.57.10.847

2. Wedzicha JA, Seemungal TA. COPD exacerbations: defining their cause and prevention. Lancet (London, England). 2007;370 (9589):786-796. doi:10.1016/S0140-6736(07)61382-8

3. Soler-Cataluna JJ, Martinez-Garcia MA, Roman Sanchez P, et al. Severe acute exacerbations and mortality in patients with chronic obstructive pulmonary disease. Thorax. 2005;60(11):925-931. doi:10.1136/thx.2005.040527

4. Christenhusz LC, Prenger R, Pieterse ME, et al. Cost-effectiveness of an intensive smoking cessation intervention for COPD outpatients. Nicotine Tobacco Res. 2012;14(6):657-663. doi:10.1093/ntr/ntr263

5. Albert RK, Connett J, Bailey WC, et al. Azithromycin for prevention of exacerbations of COPD. $N$ Engl J Med. 2011;365(8):689-698. doi:10.1056/NEJMoa1104623

6. Calverley PM, Anderson JA, Celli B, et al. Salmeterol and fluticasone propionate and survival in chronic obstructive pulmonary disease. N Engl J Med. 2007;356(8):775-789. doi:10.1056/NEJMoa063070

7. Martinez FJ, Calverley PM, Goehring UM, et al. Effect of roflumilast on exacerbations in patients with severe chronic obstructive pulmonary disease uncontrolled by combination therapy (REACT): a multicentre randomised controlled trial. Lancet (London, England). 2015;385 (9971):857-866. doi:10.1016/S0140-6736(14)62410-7

8. Tashkin D, Celli B, Kesten S, et al. Effect of tiotropium in men and women with COPD: results of the 4-year UPLIFT trial. Respir Med. 2010;104(10):1495-1504. doi:10.1016/j.rmed.2010.03.033

9. Berry MJ, Rejeski WJ, Adair NE, et al. Exercise rehabilitation and chronic obstructive pulmonary disease stage. Am J Respir Crit Care Med. 1999;160(4):1248-1253. doi:10.1164/ajrccm.160.4.9901014

10. Schembri S, Morant S, Winter JH, et al. Influenza but not pneumococcal vaccination protects against all-cause mortality in patients with COPD. Thorax. 2009;64(7):567-572. doi:10.1136/ thx.2008.106286

11. Casas A, Troosters T, Garcia-Aymerich J, et al. Integrated care prevents hospitalisations for exacerbations in COPD patients. Eur Respir J. 2006;28(1):123-130. doi:10.1183/09031936.06.00063205
12. Lisspers $\mathrm{K}$, Johansson $\mathrm{G}$, Jansson $\mathrm{C}$, et al. Improvement in COPD management by access to asthma/COPD clinics in primary care: data from the observational PATHOS study. Respir Med. 2014;108 (9):1345-1354. doi:10.1016/j.rmed.2014.06.002

13. Bourbeau J, Ford G, Zackon H, et al. Impact on patients' health status following early identification of a COPD exacerbation. Eur Respir J. 2007;30(5):907-913. doi:10.1183/09031936.00166606

14. Andersson F, Borg S, Jansson SA, et al. The costs of exacerbations in chronic obstructive pulmonary disease (COPD). Respir Med. 2002;96 (9):700-708. doi:10.1053/rmed.2002.1334

15. Sedlacek MJ, Walker C. Antibiotic resistance in an in vitro subgingival biofilm model. Oral Microbiol Immunol. 2007;22(5):333-339. doi:10.1111/j.1399-302X.2007.00366.x

16. Kucukcoskun M, Baser U, Oztekin G, et al. Initial periodontal treatment for prevention of chronic obstructive pulmonary disease exacerbations. J Periodontol. 2013;84(7):863-870. doi:10.1902/ jop.2012.120399

17. Zhou X, Han J, Liu Z, et al. Effects of periodontal treatment on lung function and exacerbation frequency in patients with chronic obstructive pulmonary disease and chronic periodontitis: a 2-year pilot randomized controlled trial. J Clin Periodontol. 2014;41 (6):564-572. doi:10.1111/jcpe. 12247

18. Jones PW, Harding G, Berry P, et al. Development and first validation of the COPD assessment test. Eur Respir J. 2009;34(3):648-654. doi:10.1183/09031936.00102509

19. Global strategy for the diagnosis, management, and prevention of Chronic Obstructive Pulmonary Disease; 2017. Available from: http://www.goldcopd.com. Accessed October 19, 2021.

20. Page RC, Eke PI. Case definitions for use in population-based surveillance of periodontitis. Periodontol. 2007;78(7 Suppl):1387-1399. doi:10.1902/jop.2007.060264

21. Quirynen M, Bollen CM, Vandekerckhove BN, et al. Full- vs. partial-mouth disinfection in the treatment of periodontal infections: short-term clinical and microbiological observations. J Dent Res. 1995;74(8):1459-1467. doi:10.1177/00220345950740080501

22. Klindworth A, Pruesse E, Schweer T, et al. Evaluation of general $16 \mathrm{~S}$ ribosomal RNA gene PCR primers for classical and next-generation sequencing-based diversity studies. Nucleic Acids Res. 2013;41(1): e1. doi:10.1093/nar/gks808

23. Callahan BJ, McMurdie PJ, Rosen MJ, et al. DADA2: high-resolution sample inference from Illumina amplicon data. Nat Methods. 2016;13(7):581-583. doi:10.1038/nmeth.3869

24. Escapa IF, Huang Y, Chen T, et al. Construction of habitat-specific training sets to achieve species-level assignment in 16S rRNA gene datasets. Microbiome. 2020;8(1):65. doi:10.1186/s40168-020-00841-w

25. Wirbel J, Zych K, Essex M, et al. Microbiome meta-analysis and cross-disease comparison enabled by the SIAMCAT machine learning toolbox. Genome Biol. 2021;22:93. doi:10.1186/s13059-02102306-1

26. Landbo C, Prescott E, Lange P, et al. Prognostic value of nutritional status in chronic obstructive pulmonary disease. Am J Respir Crit Care Med. 1999;160(6):1856-1861. doi:10.1164/ ajrccm.160.6.9902115

27. Tibshirani R. Regression shrinkage and selection via the Lasso. $J$ Royal Statis Soc. 1996;58(1):267-288. doi:10.1111/j.25176161.1996.tb02080.x

28. Carter JV, Pan J, Rai SN, et al. ROC-ing along: evaluation and interpretation of receiver operating characteristic curves. Surgery. 2016;159(6):1638-1645. doi:10.1016/j.surg.2015.12.029

29. Liu Z, Zhang W, Zhang J, et al. Oral hygiene, periodontal health and chronic obstructive pulmonary disease exacerbations. $J$ Clin Periodontol. 2012;39(1):45-52. doi:10.1111/j.1600-051X.2011.01808.x

30. Shen TC, Chang PY, Lin CL, et al. Periodontal treatment reduces risk of adverse respiratory events in patients with chronic obstructive pulmonary disease: a propensity-matched cohort study. Medicine (Baltimore). 2016;95(20):e3735. doi:10.1097/MD.0000000000003735 
31. Pérez Barrionuevo AM, Gómez Real F, Igland J, et al. Periodontal health status and lung function in two Norwegian cohorts. PLoS One. 2018;13(1):e0191410. doi:10.1371/journal.pone.0191410

32. Apessos I, Voulgaris A, Agrafiotis M, et al. Effect of periodontal therapy on COPD outcomes: a systematic review. BMC Pulm Med. 2021;21:92. doi:10.1186/s12890-021-01429-2

33. Kinane DF, Stathopoulou PG, Papapanou PN. Periodontal diseases. Nat Rev Dis Primers. 2017;3:17038. doi:10.1038/nrdp.2017.38

34. Sczepanik FSC, Grossi ML, Casati M, et al. Periodontitis is an inflammatory disease of oxidative stress: we should treat it that way. Periodontol 2000. 2020;84(1):4568. doi:10.1111/prd.12342

35. Sapey E, Yonel Z, Edgar R, et al. The clinical and inflammatory relationships between periodontitis and chronic obstructive pulmonary disease. J Clin Periodontol. 2020;47(9):1040-1052. doi:10.1111/ jcpe. 13334
36. Jönsson D, Spinell T, Vrettos A, et al. Circulating endothelial progenitor cells in periodontitis. J Periodontol. 2014;85(12):1739-1747. doi:10.1902/jop.2014.140153

37. Tonetti MS, D'Aiuto F, Nibali L, et al. Treatment of periodontitis and endothelial function. $N$ Engl $J$ Med. 2007;356(9):911-920. doi:10.1056/NEJMoa063186

38. D'Aiuto F, Parkar M, Andreou G, et al. Periodontitis and systemic inflammation control of the local infection is associated with a reduction in serum inflammatory markers. $J$ Dent Res. 2004;83 (2):156-160. doi:10.1177/154405910408300214

39. Teeuw WJ, Slot DE, Susanto H, et al. Treatment of periodontitis improves the atherosclerotic profile: a systematic review and meta-analysis. J Clin Peridontol. 2014;41(1):70-79. doi:10.1111/ jcpe. 12171

\section{Publish your work in this journal}

The International Journal of COPD is an international, peer-reviewed journal of therapeutics and pharmacology focusing on concise rapid reporting of clinical studies and reviews in COPD. Special focus is given to the pathophysiological processes underlying the disease, intervention programs, patient focused education, and self management protocols. This journal is indexed on PubMed Central, MedLine and CAS. The manuscript management system is completely online and includes a very quick and fair peer-review system, which is all easy to use. Visit http://www.dovepress.com/testimonials.php to read real quotes from published authors. 